\title{
Does the Surgical Reduction of High Grade Spondylolisthesis Restore Spino-Pelvic Alignment? An Analysis of 35 Patients
}

\author{
Rohit Amritanand, Justin Arockiaraj, Kenny S. David, Venkatesh Krishnan \\ Spinal Disorder Surgery Unit, Department of Orthopaedics, Christian Medical College \& Hospital, Vellore, India
}

\section{Study Design: Retrospective case series.}

Purpose: This study aimed to analyze how the sagittal spinopelvic alignment is influenced by an attempted surgical reduction of the L5-S1 segment in patients with high-grade spondylolisthesis (HGS).

Overview of Literature: Conventional treatment strategies stress the importance of achieving fusion across the lumbosacral junction in patients with HGS. The role of reduction in this subset of patients is controversial.

Methods: This is a retrospective case series of 35 patients with Meyerding grades III, IV, or V spondylolisthesis who underwent surgical treatment in our institution. Before and after surgery, we took standing lateral radiographs from L1 vertebra to pelvis, including the femoral heads, and measured the slip grade, pelvic incidence, sacral slope, pelvic tilt, lumbosacral angle, and lumbar lordosis. Patients were subdivided into "balanced" and "unbalanced" pelvis groups. To determine the effect and correlation of reduction on these spinopelvic parameters, we statistically compared the pre- and postoperative measurements.

Results: The average follow-up was 9 months (range, 3-169 months). Slip grade improved from an average $74.0 \% \pm 13.2 \%$ to $30.0 \% \pm 14.0 \%(p<0.001)$, and lumbosacral angle reduced from an average $32.0^{\circ} \pm 11.6^{\circ}$ to $6.0^{\circ} \pm 0.6^{\circ}(p<0.001)$. Although the pelvic tilt was reduced, this was not significant. There was a modest negative correlation between the reduction in slip grade and the increase in sacral slope $(r=-0.3, p=0.06)$. At follow-up, five patients improved, from an unbalanced pelvis to a balanced pelvis. Fusion occurred in 33 patients (95\%).

Conclusions: Surgical reduction of HGS restores the lumbosacral alignment. However, a similar trend is not noted with the pelvic parameters.

Keywords: Lumbosacral spine; High-grade spondylolisthesis; Sagittal balance; Reduction

\section{Introduction}

According to the Meyerding classification, spondylolisthesis or the anterior translation of vertebra is defined as high grade when the translation of the L5 vertebra over the sacrum is greater than $50 \%$ [1]. Most surgeons agree that there is a prominent role for surgery in the treatment of this challenging condition [2,3]. The usual indications

Received May 19, 2020; Revised Aug 10, 2020; Accepted Aug 11, 2020

Corresponding author: Rohit Amritanand

Spinal Disorders Surgery, Department of Orthopaedics, Christian Medical College \& Hospital, Ida Scudder Road, Vellore, Tamil Nadu, 632004, India

Tel: +91-416-228-2731, Fax: +91-416-228-2288, E-mail: rohit@cmcvellore.ac.in 
for surgery included progression of slip, neurological deficit, unrelenting back pain or radicular leg pain, and spinal deformity. The spinal deformity may be because of the translation of L5 over the sacrum or the secondary postural adaptations of the pelvis to the severe spondylolisthesis [4].

Conventional treatment strategies stress the importance of achieving fusion across the lumbosacral junction [5]. The role of surgical reduction continues to fuel controversy [6]. The scope of this debate extends between those who advocate in-situ fusion [7-9] and those who strive for near-complete $[3,10]$. Others suggest that reduction is indicated in patients with an unbalanced (retroverted) pelvis $[5,11]$. In most publications, the techniques of reduction and the associated complications, especially neurological deficit, have been well described [3,11-13]. There are very limited data describing the effect that this reduction of severe spondylolisthesis has on the sagittal balance of the spine and the orientation of the pelvis in the postoperative period $[5,11,14,15]$. Restoration of these spinopelvic parameters is vital because not only does it influence the resolution of the spinal deformity and listhetic posture but also it is known to be an important factor in contributing to postoperative pain even in patients who have achieved lumbosacral fusion [16].

In this study, we aimed to analyze how the sagittal spinopelvic alignment is influenced by an attempted surgical reduction of the L5-S1 segment in our patients with highgrade spondylolisthesis (HGS), with the hypotheses that surgical correction at the lumbosacral level is associated with an improvement in the shape of the spine and the orientation of the pelvis and that there is a linear correlation between the lumbosacral and pelvic parameters.

\section{Materials and Methods}

This was a retrospective study on 41 patients diagnosed with HGS based on the Meyerding classification [1]. The study was approved by the Institutional Review Board of Christian Medical College, Vellore (IRB approval no., 11411). All patients were surgically managed in the Spine Surgery Unit, Department of Orthopaedics, of large tertiary care, teaching hospital in South India from 2001 to 2015. Indications for surgery included progression of slip, neurological deficit, spinal deformity, and back pain or radicular leg pain. Patients were operated on by a posterioronly approach or combined anterior-posterior approach

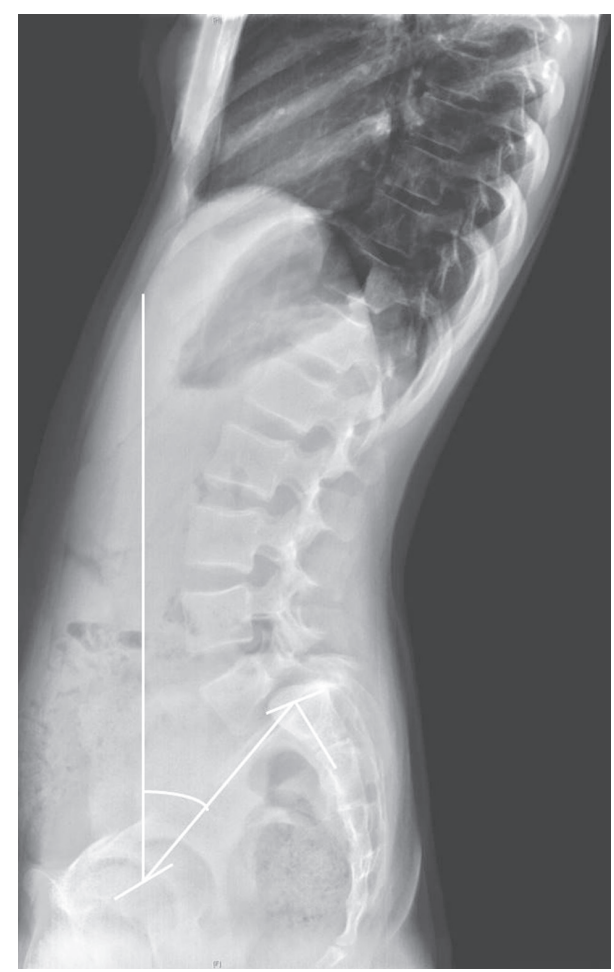

Fig. 1. Plain radiograph (standing lateral view from L1 to S1) of a 12-year-old girl whose pelvis was classified as "unbalanced" preoperatively. Her preoperative slip grade was $77 \%$, sacral slope of $30^{\circ}$, pelvic tilt of $35^{\circ}$, lumbosacral angle of $5^{\circ}$, and lumbar lordosis of $58^{\circ}$.

with reduction and interbody fusion. Before and after surgery, we took a complete set of digital radiographs including standing lateral radiographs from L1 vertebra to pelvis and the femoral heads using a digital X-ray machine (Siemens Aritos, 1,000 mA; Siemens, Munich, Germany) (Figs. 1, 2). The radiographic parameters were measured using dedicated software (Centricity Enterprise V3.0; GE Medical Systems Information Technologies Inc., Wauwatosa, WI, USA), resulting in fast and accurate calculation of the various parameters by interactive digitization of various anatomical landmarks of the spine and pelvis on a lateral radiograph. Six patients had suboptimal or incomplete radiographs and hence were excluded from the final analysis. Of the remaining 35 patients, slip grade, pelvic incidence, sacral slope (SS), pelvic tilt (PT), lumbosacral angle, and lumbar lordosis were measured on the radiograph. We performed an analysis of changes in the postprocedural spinopelvic parameters compared with the preoperative values and described the complications. According to the method described by Hresko et al. [11], we subdivided the patients into a balanced pelvis group (high SS and low PT) and an unbalanced pelvis group (low 


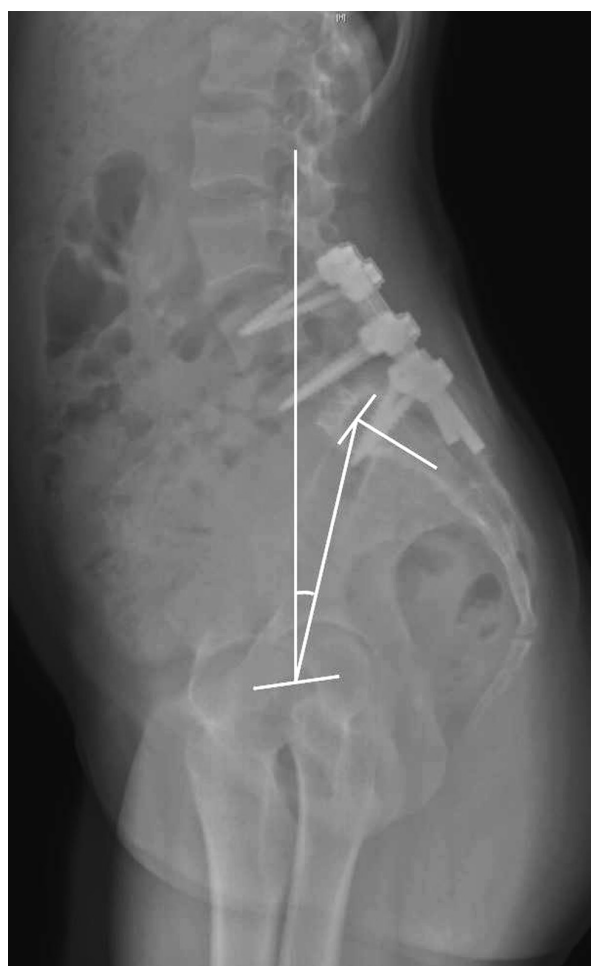

Fig. 2. At 16-month follow-up, her slip had reduced to $30 \%$. Pelvic tilt has decreased significantly and fusion mass is centered well over the hips. A good restoration of spinopelvic parameters is noted (sacral slope of $54^{\circ}$, pelvic tilt of $14^{\circ}$, lumbosacral angle of $12^{\circ}$, and lumbar lordosis of $64^{\circ}$.

SS and high PT). By this method, we can obtain the subclassification of HGS based on the version of the pelvis by plotting the SS and the PT on a graph wherein the two groups were delineated by a line $y=(0.844835 x)+25.021$, where $y$ indicates SS, and $x$ indicates PT. In addition to radiographic spinopelvic parameters, we also noted the implant and fusion status. We deemed fusion to have occurred if there was bridging trabecular bone visualized across the interbody area on a good quality lateral plain radiographs and if there was no evidence of implant failure in terms of breakage or loosening.

A total of 35 patients (29 women and six men) were included in the final analysis. The mean age of the patients was 27 years (range, 7-50 years). According to the Meyerding classification system, the cohort included 16 patients with grade III, 15 patients with grade IV, and four patients with grade $\mathrm{V}$ developmental spondylolisthesis. The average slip grade was $74 \%$ (range, $54 \%-100 \%$ ), and the average pelvic incidence was $61^{\circ}$ (range, $37^{\circ}-84^{\circ}$ ). All patients were treated with posterior instrumentation using pedicle screws. In 20 patients, the instrumentation was limited to the L5-S1 segment, whereas in the remaining
15 patients, it was extended up to L4 vertebra at the discretion of the surgeon. In the majority of patients $(n=28)$, fusion was performed through a posterior approach, whereas the remaining seven patients underwent a staged anterior transperitoneal release and anterior interbody fusion.

\section{Surgical technique}

For patients who underwent the posterior-only approach, a midline skin incision was made, and subperiosteal dissection was performed up to the tips of the L5 transverse process. Pedicle screws were placed bilaterally in L5 and $\mathrm{S} 1$ using $\mathrm{C}$-arm guidance. A wide laminectomy of $\mathrm{L} 5$ was performed, and the exiting L5 nerve roots were visualized and well decompressed. The disk space was identified, and discectomy was performed. Using an osteotome, we excised the dome of S1, and we osteotomized the anterior beak of L5 under C-arm guidance. In monosegmental surgery, the rods were fixed, and the L5 vertebra was sequentially reduced. In patients where the surgeon decided to extend the instrumentation to L4, depending on the adequacy of screw purchase, the rods were fixed and distracted to assist translation and reduction. Once reduction was achieved, a meshed cage with local morselized cortico-cancellous autograft was placed into the disc space, and the rods were compressed. In the early part of the study (2001-2005), combined anterior-posterior procedures were performed. An access surgeon provided anterior transperitoneal exposure to the L5-S1 disc space, then a discectomy and release was performed, and the patient was turned prone for the posterior instrumented reduction and fusion as described. After 2005, with our growing experience and access to better instrumentation, we began performing the procedure through a posterioronly approach.

\section{Statistical methods}

Categorical data were expressed as percentages, and continuous data were expressed as mean (standard deviation) if normal or median (interquartile range) if skewed. The pre- and post-differences in radiographic parameters were measured using paired $t$-test if the data are normal or Wilcoxon-signed rank test if the data are skewed. The radiographic parameter values among the balanced and unbalanced groups were compared using an independent 
$t$-test if the data are normal and Mann-Whitney $U$-test if the data are skewed. The correlation between change in slip grade and change in the other parameters were analyzed using Pearson's correlation coefficient $(r)$. The analysis was performed using intercooled Stata statistical software ver. 13.1 (Stata Corp., College Station, TX, USA) at a significant level of $p<0.05$.

\section{Results}

The average follow-up of the patients was 39 months

Table 1. Changes in the radiological parameters measured in the overall study group

\begin{tabular}{lccc} 
Radiographic variable & Preoperative & Postoperative & $p$-value \\
\hline Slip grade $(\%)$ & $73.0 \pm 13.2$ & $30.0 \pm 14.6$ & $<0.001^{* * *}$ \\
\hline Sacral slope $\left(^{\circ}\right)$ & $35.3 \pm 16.5$ & $35.94 \pm 14.8$ & 0.77 \\
\hline Pelvic tilt $\left({ }^{\circ}\right)$ & $24.8 \pm 11.7$ & $23.09 \pm 11.4$ & 0.40 \\
\hline Lumbo-sacral angle $\left(^{\circ}\right)$ & $32.0(11.6)$ & $6(0.60)$ & $<0.001^{* * *}$ \\
\hline Lumbar lordosis $\left(^{\circ}\right)$ & $54.3 \pm 14.0$ & $41.7 \pm 14.3$ & $<0.001^{* * *}$
\end{tabular}

Values are presented as mean \pm standard deviation or median (interquartile range).

${ }^{* * *} p<0.001$. (range, 3-163 months). Thirty-three patients (95\%) achieved solid fusion. Of the six patients with neurological deficits, two patients had cauda equina syndrome, and four patients had the Medical Research Council (MRC) grades II and III power of L5 nerve root. Of these, four patients with nerve root deficits improved by two MRC grades. The two patients with preoperative cauda equina syndrome did not improve at the final follow-up. Table 1 provides mean and standard deviations for all the radiological variables measured before surgery and at last available follow-up. As shown in the table, slip grade, lumbosacral angle, and lumbar lordosis improved significantly, whereas SS and PT have no significant changes. Slip grade improved from an average $74.0 \% \pm 13.2 \%$ to $30.0 \% \pm 14.0 \%$ $(p<0.001)$, and lumbosacral angle reduced from an average $32.0^{\circ} \pm 11.6^{\circ}$ to $6.0^{\circ} \pm 0.6^{\circ}(p<0.001)$.

After classifying the patients according to the version of the pelvis (Table 2), 17 patients were categorized in the balanced pelvis group and 18 patients in the unbalanced pelvis group. The surgical reduction improved the pelvic version in 16 patients (90\%) with an unbalanced pelvis (Fig. 2); however, this did not approach statistical significance. The reduction, however, did have a very significant

Table 2. Changes in the radiological parameters measured in the balanced and unbalanced pelvis group

\begin{tabular}{|c|c|c|c|c|}
\hline Radiographic variable & Balanced pelvis & $p$-value & Unbalanced pelvis & $p$-value \\
\hline Sacral slope & & 0.06 & & 0.12 \\
\hline Preop & $48.7 \pm 9.20$ & & $22.6 \pm 10.7$ & \\
\hline Postop & $44.3 \pm 10.5$ & & $28.0 \pm 14.1$ & \\
\hline Pelvic tilt & & 0.88 & & 0.29 \\
\hline Preop & $17.6 \pm 5.0$ & & $31.6 \pm 12.22$ & \\
\hline Postop & $17.9 \pm 7.7$ & & $27.9 \pm 12.4$ & \\
\hline Lumbosacral angle & & $<0.001$ & & $<0.001$ \\
\hline Preop & $11.0(5.00$ to 34.0$)$ & & 46.5 (32 to 66$)$ & \\
\hline Postop & 0.0 (0.00 to 2.0$)$ & & $0.0(-2.00$ to 14.0$)$ & \\
\hline Lumbar lordosis & & 0.004 & & 0.01 \\
\hline Preop & $56.5 \pm 13.0$ & & $52.2 \pm 14.8$ & \\
\hline Postop & $44.2 \pm 13.6$ & & $39.2 \pm 14.9$ & \\
\hline
\end{tabular}

Values are presented as mean \pm standard deviation or median (interquartile range). Bold type is considered statistically significant.

Preop, preoperative; Postop, postoperative.

Table 3. Correlation between changes in slip grade and pelvic parameters

\begin{tabular}{|c|c|c|c|}
\hline Variable & Overall $(n=35)$ & Balanced $(n=17)$ & Unbalanced $(n=18)$ \\
\hline Change in slip grade vs. change in sacral slope & $-0.32(0.06)$ & $-0.07(0.79)$ & $-0.25(0.31)$ \\
\hline Change in slip grade vs. change in pelvic tilt & $0.12(0.48)$ & $0.13(0.60)$ & $-0.01(0.98)$ \\
\hline
\end{tabular}


improvement in the alignment of the spine in this subgroup. The lumbosacral slip angle was reduced from $46.5^{\circ}$ to $0^{\circ}(p<0.001)$. A similar trend was noted in the change in lumbar lordosis (Table 2). At follow-up, five patients changed to a balanced pelvis group from the unbalanced pelvis group.

Furthermore, there was a negative correlation between the change in slip grade (i.e., reduction) and the change in SS across the entire cohort. This was not statistically significant. There was no correlation between changes in slip grade and pelvic parameters (Table 3 ).

We noted complications in eight patients (22\%). Among them, two patients have implant failure, with one patient demonstrating an immediate loss of reduction in the postoperative period. The patient was advised a revision surgery; however, the patient did not undergo the procedure. The other patient had an L5 screw breakage but progressed to fusion. Five patients developed neurological deficits in the form of L5 root injury. Among them, four patients with transient deficits (MRC grades 0-IV) demonstrated complete improvement within 6 months of follow-up, and the other patient continued to have grade 3 weakness of left L5 nerve roots at last follow-up. Another patient had a wound infection that required surgical debridement and antibiotic administration.

\section{Discussion}

The present study provides a critical analysis of sagittal spinopelvic alignment before and after surgical correction of HGS. Our goal was to determine if the reduction of the spondylolisthesis had an impact on the alignment of the spine through the reduction of lumbosacral angulation and lumbar lordosis and also on the pelvic version.

We found that the alignment of the spine significantly improved both in terms of the correction of the lumbosacral kyphosis and the restoration of the lumbar lordosis. A similar effect was, however, not noted in the orientation of the pelvis. Previous studies including the one by Hresko et al. [5] also observed this trend [10,14,17,18] (Table 4). They suggested that the achievement of fusion in these patients was a more important determinant of outcome than correction of the pelvic parameters. It is known that the reduction of the slip grade increases the surface area of bone contact and the correction of lumbosacral angulation reduces the high shear forces that exist across the lumbosacral junction [3]. Our study confirmed that con-

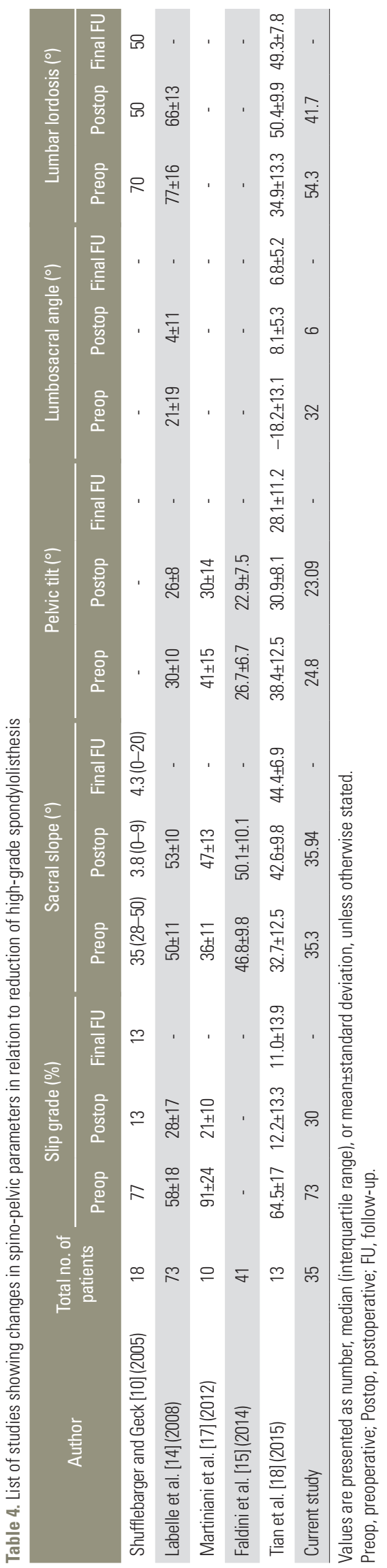


Table 5. Changes in spino-pelvic parameters of patients treated with 'in-situ' fusion

\begin{tabular}{|c|c|c|c|c|c|c|c|c|c|c|c|}
\hline \multirow{2}{*}{ Author } & \multirow{2}{*}{$\begin{array}{c}\text { Total no. of } \\
\text { patients }\end{array}$} & \multicolumn{2}{|c|}{ Slip grade (\%) } & \multicolumn{2}{|c|}{ Sacral slope $\left({ }^{\circ}\right)$} & \multicolumn{2}{|c|}{ Pelvic tilt $\left({ }^{\circ}\right)$} & \multicolumn{2}{|c|}{ Lumbosacral angle $\left({ }^{\circ}\right)$} & \multicolumn{2}{|c|}{ Lumbar lordosis $\left({ }^{\circ}\right)$} \\
\hline & & Preop & Final FU & Preop & Final FU & Preop & Final FU & Preop & Final FU & Preop & Final FU \\
\hline Lamberg et al. [7] (2007) & 25 & 71 (54 to 100) & 70 (43 to 100$)$ & - & - & - & - & $22.0 \pm 15.1$ & $20.5 \pm 16.2$ & $59.3 \pm 15.3$ & $65.7 \pm 15.5$ \\
\hline Jalanko et al. [21] (2011) & 19 Children & $69.5(51-107)$ & $55.6(23-89)$ & - & - & - & - & - & - & $60.7(26-85)$ & $72.9(50-96)$ \\
\hline Jalanko et al. [21] (2011) & 19 Adults & $66.7(53-93)$ & $65.2(45-87)$ & - & - & - & - & - & - & $66.2(50-97)$ & $69.6(48-93)$ \\
\hline Martiniani et al. [17] (2012) & 6 & $79 \pm 12$ & $75 \pm 17$ & $54 \pm 18$ & $55 \pm 16$ & $25 \pm 13$ & $24 \pm 12$ & - & - & - & - \\
\hline
\end{tabular}

Values are presented as number, median (interquartile range), or mean \pm standard deviation, unless otherwise stated.

Preop, preoperative; Postop, postoperative; FU, follow-up.

trolled reduction reduces the slip grade and lumbosacral kyphosis, significantly setting up an environment conducive for osseous fusion.

One of the major hazards of reduction is the increased rate of L5 root injury reported [19]. In our series, we noted this in five patients (14\%). Many strategies including avoiding excessive distraction and correction of the pelvic retroversion that relieves tension on the sacral roots have been reported to be effective in reducing this major complication [3]. In addition to instrumentation-based reduction, maneuvers like a hyperextension of the hips have been recommended by Ruf et al. [3] to facilitate correction of the retroversion. Intraoperative neurological monitoring is a useful tool during this procedure [20].

Previous studies have recommended in-situ fusion [7-9]. Despite some reports of good outcomes by this technique [7,17,21] (Table 5), in-situ fusion has some serious disadvantages [3], such as pseudarthrosis [22], the progression of deformity due to abnormal sagittal profile [9], and neurological compromise as late sequelae [23]. Therefore, reduction may reduce these undesirable effects.

Restoration of spinopelvic alignment is also important in relation to the biomechanics and normal functioning of the spine. Previous studies have demonstrated that spinopelvic parameters impact health-related quality of life in the adult deformity population. Lazennec et al. [16] analyzed the relationship between sagittal spinopelvic alignment and postoperative pain in patients undergoing lumbosacral fusion and found that patients with a higher PT had more back pain despite fusion. They suggested that achieving a state of fusion should not be the only goal of a lumbosacral fusion. Proper positioning of the vertebra was also important to minimize back pain in the postoperative period [16]. A positive sagittal vertical axis (i.e., and anterior shift of the C7 plumb line) and loss of lum- bar lordosis have been shown to impact pain and function scores negatively $[24,25]$. Because the major contribution to lumbar lordosis (approximately 66\%) comes from the L4-5 and L5-S1 motion segments [26], it is imperative to achieve adequate lordosis when incorporating these segments in a lumbar fusion to restore a physiological sagittal profile. A failure to regain lordosis between L4 and S1 can trigger compensatory mechanisms such as hyperextension in the motion segments immediately above the fusion, along with retroversion of the pelvis (increasing PT). This finding stresses the importance of analyzing the effect that surgical reduction has on the restoration of spinopelvic parameters.

The strengths of the study were the fact that the majority of the patients were available for long-term follow-up with an average of 3 years and 4 months. This permitted an adequate duration of time for the necessary postural adaptations to set in. Also, given that this study was performed in a single academic institution, the indications, approach, and surgical techniques were fairly uniform despite the long period (15 years) of study. The retrospective nature introduces some inherent limitations. Incomplete data in the form of suboptimal radiographs in six patients necessitated their exclusion from the analysis. We recognize that the clinical impact of the measured changes has not been analyzed. Given the modest improvement in pelvic parameters, our study demonstrates it would be important to study what effect this has on the patient. We anticipate that this may form the basis of a well-designed prospective study where validated clinical outcome tools, such as the Oswestry Disability Index and 36-item Short Form Health Survey questionnaires, may be used to assess the impact of the restoration of spinopelvic balance on clinical outcomes. 


\section{Conclusions}

Surgical reduction of HGS restores the lumbosacral alignment. However, a similar trend is not noted with the pelvic parameters. These findings indicate avenues for further research on how these radiological changes impact clinical outcomes.

\section{Conflict of Interest}

No potential conflict of interest relevant to this article was reported.

\section{Acknowledgments}

The authors wish to acknowledge Dr. N. Guruswamy for his assistance with data collection and Mrs. S. Mahasampath Gowri for her assistance with the statistical analysis.

\section{Author Contributions}

Rohit Amritanand: design, analysis, interpretation of data, and draft manuscript; Justin Arockiaraj: correction, critical revision, and final approval of the draft; Kenny S. David: correction, critical revision, and final approval of the draft; and Venkatesh Krishnan: correction, critical revision, supervision, and final approval of the draft.

\section{References}

1. Meyerding HW. Spondylolisthesis. Surg Gynecol Obstet 1932;54:371-7.

2. Moller H, Hedlund R. Surgery versus conservative management in adult isthmic spondylolisthesis: a prospective randomized study: part 1 . Spine (Phila Pa 1976) 2000;25:1711-5.

3. Ruf M, Koch H, Melcher RP, Harms J. Anatomic reduction and monosegmental fusion in high-grade developmental spondylolisthesis. Spine (Phila Pa 1976) 2006;31:269-74.

4. Dubousset J. Treatment of spondylolysis and spondylolisthesis in children and adolescents. Clin Orthop Relat Res 1997;337:77-85.

5. Hresko MT, Hirschfeld R, Buerk AA, Zurakowski $D$. The effect of reduction and instrumentation of spondylolisthesis on spinopelvic sagittal alignment. J Pediatr Orthop 2009;29:157-62.
6. Passias PG, Poorman CE, Yang S, et al. Surgical treatment strategies for high-grade spondylolisthesis: a systematic review. Int J Spine Surg 2015;9:50.

7. Lamberg T, Remes V, Helenius I, Schlenzka D, Seitsalo S, Poussa M. Uninstrumented in situ fusion for high-grade childhood and adolescent isthmic spondylolisthesis: long-term outcome. J Bone Joint Surg Am 2007;89:512-8.

8. Peek RD, Wiltse LL, Reynolds JB, Thomas JC, Guyer DW, Widell EH. In situ arthrodesis without decompression for grade-III or IV isthmic spondylolisthesis in adults who have severe sciatica. J Bone Joint Surg Am 1989;71:62-8.

9. Seitsalo S, Osterman K, Hyvarinen H, Schlenzka D, Poussa M. Severe spondylolisthesis in children and adolescents: a long-term review of fusion in situ. J Bone Joint Surg Br 1990;72:259-65.

10. Shufflebarger HL, Geck MJ. High-grade isthmic dysplastic spondylolisthesis: monosegmental surgical treatment. Spine (Phila Pa 1976) 2005;30:S42-8.

11. Hresko MT, Labelle H, Roussouly P, Berthonnaud E. Classification of high-grade spondylolistheses based on pelvic version and spine balance: possible rationale for reduction. Spine (Phila Pa 1976) 2007;32:2208-13.

12. Smith JA, Deviren V, Berven S, Kleinstueck F, Bradford DS. Clinical outcome of trans-sacral interbody fusion after partial reduction for highgrade 15-s1 spondylolisthesis. Spine (Phila Pa 1976) 2001;26:2227-34.

13. Ilharreborde B, Fitoussi F, Morel E, Bensahel H, Pennecot GF, Mazda K. Jackson's intrasacral fixation in the management of high-grade isthmic spondylolisthesis. J Pediatr Orthop B 2007;16:16-8.

14. Labelle H, Roussouly P, Chopin D, Berthonnaud E, Hresko T, O'Brien M. Spino-pelvic alignment after surgical correction for developmental spondylolisthesis. Eur Spine J 2008;17:1170-6.

15. Faldini C, Di Martino A, Perna F, Martikos K, Greggi T, Giannini S. Changes in spino-pelvic alignment after surgical treatment of high-grade isthmic spondylolisthesis by a posterior approach: a report of 41 cases. Eur Spine J 2014;23 Suppl 6:714-9.

16. Lazennec JY, Ramare S, Arafati N, et al. Sagittal alignment in lumbosacral fusion: relations between radiological parameters and pain. Eur Spine J 2000;9:4755. 
17. Martiniani M, Lamartina C, Specchia N. "In situ" fusion or reduction in high-grade high dysplastic developmental spondylolisthesis (HDSS). Eur Spine J 2012;21 Suppl 1:S134-40.

18. Tian W, Han XG, Liu B, et al. Posterior reduction and monosegmental fusion with intraoperative threedimensional navigation system in the treatment of high-grade developmental spondylolisthesis. Chin Med J (Engl) 2015;128:865-70.

19. Petraco DM, Spivak JM, Cappadona JG, Kummer FJ, Neuwirth MG. An anatomic evaluation of L5 nerve stretch in spondylolisthesis reduction. Spine (Phila Pa 1976) 1996;21:1133-8.

20. Mizuno K, Mikami Y, Nagae M, et al. Instrumented reduction and monosegmental fusion for Meyerding grade IV developmental spondylolisthesis: a report of 3 cases. Medicine (Baltimore) 2014;93:e244.

21. Jalanko T, Helenius I, Remes V, et al. Operative treatment of isthmic spondylolisthesis in children: a longterm, retrospective comparative study with matched cohorts. Eur Spine J 2011;20:766-75.
22. Roca J, Ubierna MT, Caceres E, Iborra M. One-stage decompression and posterolateral and interbody fusion for severe spondylolisthesis: an analysis of 14 patients. Spine (Phila Pa 1976) 1999;24:709-14.

23. Maurice HD, Morley TR. Cauda equina lesions following fusion in situ and decompressive laminectomy for severe spondylolisthesis: four case reports. Spine (Phila Pa 1976) 1989;14:214-6.

24. Glassman SD, Bridwell K, Dimar JR, Horton W, Berven S, Schwab F. The impact of positive sagittal balance in adult spinal deformity. Spine (Phila Pa 1976) 2005;30:2024-9.

25. Glassman SD, Berven S, Bridwell K, Horton W, Dimar JR. Correlation of radiographic parameters and clinical symptoms in adult scoliosis. Spine (Phila Pa 1976) 2005;30:682-8.

26. Bernhardt M, Bridwell KH. Segmental analysis of the sagittal plane alignment of the normal thoracic and lumbar spines and thoracolumbar junction. Spine (Phila Pa 1976) 1989;14:717-21. 